\title{
Drug Company Contact
}

National Cancer Institute

\section{Source}

National Cancer Institute. Drug Company Contact. NCI Thesaurus. Code C51822.

A person who acts as a channel for communication with a commercial enterprise which produces substances intended for use in the diagnosis, cure, mitigation, treatment, or prevention of disease. 